\title{
The Neurobiology of Maternal Mental Illness: Current understanding and future directions
}

\author{
Jodi L. Pawluski ${ }^{1}$ \\ Published online: 8 May 2019 \\ (C) Springer-Verlag GmbH Austria, part of Springer Nature 2019
}

A growing number of women suffer from peripartum mood or anxiety disorders, yet our understanding of the neurobiology of these disorders is in its infancy. We know that these mental illnesses severely affect the mother and can have pervasive effects on the child. Given that these are mental disorders, it is disappointing to note the scarcity of research investigating the neurobiological factors that are mediating maternal mental illness. The Topical Collection titled The Neurobiology of Maternal Mental Illness stemmed from a minisymposium at the Society for Neuroscience annual meeting in 2017 where the authors of the papers in the collection were brought together to talk about their research on maternal mental illness and its relation to changes in the maternal brain.

This Topical Collection takes a translational approach with reviews of the literature in both human and animal models. In addition, we aim to discuss treatment effects of pharmacotherapy on the maternal brain, although this is less well studied, with a short communication investigating the effects of SSRIs on neural biomarkers in pregnant women.

The manuscript in this series by Barba-Muller and colleagues reviews the literature of brain structural and functional changes in women during the peripartum period. The authors discuss the dynamic changes in the maternal brain that are adaptive, relating to maternal attachment and maternal care. They also highlight that this heightened peripartum neuroplasticity may increase vulnerability for development of peripartum mental illnesses.

This article is part of the Topical Collection on Neurobiology of Maternal Mental Illness

Guest Editor: Jodi Pawluski

Jodi L. Pawluski

j.pawluski@gmail.com

1 Univ Rennes, Inserm, EHESP, Irset (Institut de Recherche en Santé, Environnement et Travail), UMR_S 1085, F-35000 Rennes, France
Klampfl and Bosch continue the theme of the neurobiology of maternal care-giving with a focus on the role of corticotropic-releasing factor (CRF) in maternal neglect and anxiety. In particular, they focus on CRF effects in bed nucleus of the stria terminalis (BNST), a brain region central to maternal care-giving behaviors. As virtually no research in this area is done in humans, this article takes a translational approach and provides valuable information for neural mechanisms possibly mediating neglect or even filicide, as well as areas to focus future research for therapeutic approaches to maternal mental illness.

The review of Post and Leuner highlights the maternal reward system and its role in postpartum depression (PPD) where often the mother does not find the infant rewarding. This review focuses on the mesolimbic dopamine system which plays a key role in maternal care-giving, motivation, and mood. Research is beginning to show that this system plays a pivotal role in PPD, and together with the interaction between dopamine and oxytocin, these factors may be regulating mood and maternal care-giving behaviors in women. Again, another area for future research and potential for therapeutic approaches.

The review of Lonstein moves on to review the serotonergic system of the maternal brain. The first-line pharmacotherapies for peripartum mood and anxiety disorders are generally the selective-serotonin reuptake inhibitors (SSRIs); however, Lonstein points out that we know 'very little about the typical, let alone that atypical, changes' that occur in the central serotonergic system of the maternal brain. This review points to a need to increase our understanding of the serotonergic system of the maternal brain which will lead to better and more precise pharmacotherapies.

The final piece of the Topical Collection is a short communication by Pawluski and colleagues which shows that SSRIs can increase serum levels of brain-derived neurotrophic factor (BDNF) in late pregnant women and that maternal depressive symptoms are associated with serum S100 calcium binding protein B (S100B) levels but only in 
SSRI-treated women. This is an interesting first study showing that SSRIs may interact with neural biomarkers of peripartum depression.

Together, these articles highlight key areas for future research on the neurobiology of peripartum mood and anxiety disorders. Increased understanding of the neural mech- anisms mediating the diverse peripartum affective states is needed to effectively treat and, ultimately, prevent these debilitating diseases.

Publisher's Note Springer Nature remains neutral with regard to jurisdictional claims in published maps and institutional affiliations. 